\title{
Ferrous Ferric Chloride Stimulates the Skin Cell Function and Hair Growth in Mice
}

\author{
Tomohisa HiroBE ${ }^{a, b}$ \\ ${ }^{a}$ Radiation Effect Mechanism Research Group, National Institute of Radiological Sciences; Anagawa 4-9-1, Inage-ku, \\ Chiba 263-8555, Japan: and ${ }^{b}$ Graduate School of Science, Chiba University; Yayoi-cho 1-33, Inage-ku, Chiba 263-8522, \\ Japan. Received April 22, 2009; accepted June 3, 2009; published online June 4, 2009
}

Ferrous ferric chloride $\left(\right.$ FFC $\left.^{\circledR}\right)$ is a distinct form of aqueous iron composed of a complex of ferrous chloride and ferric chloride that participates in both oxidation and reduction reactions. The author's previous study showed that the supplementation of culture medium with FFC stimulated the proliferation and differentiation of keratinocytes and melanocytes in newborn mice. FFC also stimulated the proliferation of cultured human keratinocytes, fibroblasts, and melanocytes. However, it is not known whether FFC stimulates the proliferation and differentiation of mammalian skin cells as well as hair growth in vivo. To answer this question, FFC-containing skin lotions (FFC Super Essence Plain ${ }^{\circledR}$ and Moisture ${ }^{\circledR}$ Type, Akatsuka Co.) were painted on the dorsal skin of newborn C57BL/10JHir (B10) mice and tested for their proliferation- and differentiation-stimulating effects on keratinocytes, fibroblasts, and melanocytes as well as for their stimulating effects on the hair growth. This treatment stimulated the proliferation and differentiation of epidermal keratinocytes, dermal fibroblasts, and epidermal and dermal melanocytes in the skin as well as hair growth. From 2 to 3 weeks after birth B10 mice generally lose their hairs except those on the head at the telogen stage of the hair growth cycle due to the expression of the alopecia. The treatment with FFC lotions markedly inhibited the alopecia hair-loss. These results suggest that FFC can stimulate the proliferation and differentiation of keratinocytes, fibroblasts, and melanocytes in the skin as well as the hair growth, and, in addition, can inhibit the alopecia hair-loss.

Key words ferrous ferric chloride; keratinocyte; fibroblast; melanocyte

Ferrous ferric chloride ( $\mathrm{FFC}^{\circledR}$; Akatsuka Co., Mie, Japan) is a distinct form of aqueous iron composed of a complex of ferrous chloride and ferric chloride that participates in both oxidation and reduction reactions. ${ }^{1)}$ However, the mechanism by which $\mathrm{Fe}(\mathrm{II})$ and $\mathrm{Fe}(\mathrm{III})$ contained in FFC affects living organisms is not fully understood. FFC elicits specific biological effects such as stimulating plant growth, especially root growth. ${ }^{2)}$ In animals, FFC also increases the red blood cell number and hemoglobin content in rats. ${ }^{3)}$ The author's previous study showed that FFC stimulated the proliferation of cultured keratinocytes, melanoblasts, and melanocytes derived from newborn mice. ${ }^{4)}$ Moreover, FFC stimulated the differentiation of murine keratinocytes and melanocytes. ${ }^{5)}$ The proliferation of keratinocytes, melanoblasts, and melanocytes was stimulated to the same extent (a 2-fold increase), suggesting that the proliferation of keratinocytes and melanocytes in the mouse epidermis may be equally affected by FFC. Thus, it is possible that FFC activates skin function by promoting cell renewal through the stimulation of the proliferation and differentiation of both keratinocytes and melanocytes. The author's previous study also showed that FFC stimulated the proliferation of cultured human keratinocytes, melanocytes, and fibroblasts. ${ }^{6}$ However, it is not known whether FFC can stimulate the proliferation and differentiation of mammalian keratinocytes, fibroblasts, melanocytes in vivo. It is also not known whether FFC can stimulate the hair growth in vivo. Although the three-dimensional structure of hair is different from that of the skin, hair consists of the same cells as the skin, namely keratinocytes, fibroblasts, melanocytes. ${ }^{\text {) }}$

FFC-containing skin lotions may be useful in answering these questions. Various kinds of plant-derived moisturizers are included in these skin lotions in addition to FFC. The moisturizers present in FFC lotions prevent evaporation of water, thereby allowing FFC to resist dysfunction for a long period. FFC Super Essence Plain Type ${ }^{\circledR}$ (FFC Plain; Akatsuka) contains FFC water, various kinds of plant-derived moisturizing factors, and various kinds of plant extracts containing skin-activating factors. The other type of FFC lotion, FFC Super Essence Moisture Type ${ }^{\circledR}$ (FFC Moisture; Akatsuka) contains 3 kinds of oils such as rose oil, grape seed oil, and jojoba oil as well as emulsifiers in addition to the ingredients contained in FFC Plain. In this study, to investigate the effects of FFC on murine skin and hair, FFC Plain and Moisture were painted on the dorsal skin of neonatal mice and tested for their proliferation- and differentiation-stimulating effects on keratinocytes, fibroblasts, melanocytes, and for their effects on hair growth and the alopecia hair-loss.

\section{MATERIALS AND METHODS}

Mice All animals used in this study were house mice (Mus musculus) of strain C57BL/10JHir (B10). They were given water and a commercial diet, OA-2 (Clea Japan, Tokyo, Japan) ad libitum. They were maintained at $24 \pm 1{ }^{\circ} \mathrm{C}$ with $40-60 \%$ relative humidity, and $12 \mathrm{~h}$ of fluorescent light was provided daily. This study was approved by the ethics committee of the National Institute of Radiological Sciences in accordance with the guidelines of the National Institute of Health.

Skin Experiments $\mathrm{FFC}$ was prepared as follows. ${ }^{1)}$ Ferric chloride $\left(\mathrm{FeCl}_{3} \cdot 6 \mathrm{H}_{2} \mathrm{O}\right)$ was dissolved in a mixed solution of $2 \mathrm{~m}$ ammonium formate, $1 \mathrm{~m}$ hydroxylamine, and $1 \mathrm{~m}$ formamide to a final concentration of $1 \mathrm{M}$ and then diluted with $\mathrm{H}_{2} \mathrm{O}$ to $10^{-8}-10^{-14} \mathrm{~mm}$. An additional $1 \mathrm{~g}$ of $\mathrm{FeCl}_{3} \cdot 6 \mathrm{H}_{2} \mathrm{O}$ per $10 \mathrm{ml}$ solution was dissolved in the solution, which was then gradually heated to $100^{\circ} \mathrm{C}$ until FFC could be crystallized. The proportion of $\mathrm{Fe}(\mathrm{II})$ to $\mathrm{Fe}(\mathrm{III})$ was $4: 6\left(10^{-8} \mathrm{~mm}\right.$ 
solution), $6: 4\left(10^{-12} \mathrm{~mm}\right.$ solution), and $7: 3\left(10^{-14} \mathrm{~mm}\right.$ solution). Analysis using ion-exchange chromatography and $\mathrm{X}$ ray diffractometry suggested that $\mathrm{Fe}(\mathrm{II})$ and $\mathrm{Fe}(\mathrm{III})$ formed dimers.

FFC Plain and Moisture were kind gift from Akatsuka and were painted $(0.25 \mathrm{ml})$ every day on the dorsal skin of B10 mice from $0.5 \mathrm{~d}$ after birth. At the same time, pieces of untreated skin were excised from the dorsal side of the animals and fixed in Bouin's fixative for $16-18 \mathrm{~h}$ at room temperature or in $16 \%$ formalin (Wako Pure Chemical Industries, Osaka, Japan) in phosphate buffer (Wako; $\mathrm{pH}$ 7.0) for 16$18 \mathrm{~h}$ at $4^{\circ} \mathrm{C}$. After the treatment with FFC Plain and Moisture, pieces of skin were also excised from the dorsal side of the animals at 2.5 and $5.5 \mathrm{~d}$ and fixed similarly. Untreated (control) skin samples were similarly fixed at 2.5 and $5.5 \mathrm{~d}$. Each experimental and control group consisted of 3 mice and each experiment was repeated 3 times. Each of 9 skin samples was processed into histological and histochemical sections. Skin samples fixed with Bouin's fixative were processed into paraffin (Merck kGaA, Darmstadt, Germany) sections ( $8 \mu \mathrm{m}$ thick), and stained with hematoxylin (Merck) and eosin (Merck). The number of mitotic keratinocytes was counted per unit surface area of the epidermis, and the index of mitotic activity was expressed as the number of mitotic keratinocytes $/ 0.1 \mathrm{~mm}^{2}$ epidermis. The method for determining the mitotic index of fibroblasts was as follows. The total number of fibroblasts was measured for each skin sample. The number of fibroblasts and mitotic fibroblasts were counted per area of a rectangle between hair follicles and from the basement membrane to a depth of approximately $100 \mu \mathrm{m}$. The mitotic index (\%) was estimated by calculating the proportion of mitotic fibroblasts to the total number of fibroblasts.

Skin samples fixed in $16 \%$ formalin in phosphate buffer were washed with distilled water and incubated with $0.1 \% \mathrm{~L}-$ dihydroxyphenylalanine (dopa; Wako) solution in phosphate buffer ( $\mathrm{pH} 7.4$ ) for $16-18 \mathrm{~h}$ at $37^{\circ} \mathrm{C}$. The dopa reaction detects tyrosinase-containing differentiated melanocytes. ${ }^{8)}$ After the dopa reaction, skin samples were processed to paraffin sections ( $10 \mu \mathrm{m}$ thick), and stained with ammoniacal silver nitrate solution (Wako; combined dopa-premelanin reaction). ${ }^{9,10)}$ The combined dopa-premelanin reaction preferentially reveals undifferentiated melanoblasts that contain stage I and II melanosomes in addition to tyrosinase-containing differentiated melanocytes. The ammoniacal silver nitrate reaction specifically reveals unmelanized stage I and II melanosomes as well as melanized melanosomes in melanocytes, the metallic silver particles being deposited with a high degree of selectivity. ${ }^{11,12)}$ The sections were counterstained with eosin (Merck). The number of melanocytes (cells positive to the dopa reaction) as well as the number of stage I and II melanosomes-containing melanoblasts and differentiated melanocytes were counted per $0.1 \mathrm{~mm}^{2}$ of the epidermis and dermis. The number of melanoblasts was calculated by subtracting the number of melanocytes from the total number of melanoblasts plus melanocytes. ${ }^{13)}$ Melanocytes are present in both epidermis and dermis in addition to hair follicles. Since the characters of melanocytes are known to be different between epidermis and dermis, ${ }^{14}$ the number of epidermal and dermal melanocytes was counted separately to know whether the re- sponse to FFC is different between them.

Effects of FFC on Hair Growth and Hair-Loss FFC Plain and Moisture $(0.25 \mathrm{ml})$ were painted on the dorsal skin of B10 mice 4 times per week starting $0.5 \mathrm{~d}$ after birth and their effects on hair growth and the alopecia hair-loss were studied. Each experimental and control group consisted of 6-7 mice and each experiment was repeated 3-6 times. The effects of FFC Plain and Moisture on hair growth and hair-loss in each of 18 or 42 mice were determined by visual inspection and photographed. The observations of alopecia continued $21 \mathrm{~d}$ after birth. Since the effects of FFC were greater in the earlier $(0.5 \mathrm{~d})$ treatment than in the later treatment, neonatal mice were used for studying the effects of FFC on the alopecia hair-loss.

The statistical significance of the differences in the numbers of mitotic keratinocytes, fibroblasts, melanocytes, melanoblasts, and melanoblasts plus melanocytes as well as in the mitotic index of fibroblasts was determined using Student's $t$-test for comparison of groups of equal size.

\section{RESULTS}

Effects of FFC Lotions on Keratinocytes and Fibroblasts The dorsal skin of B10 mice was painted every day with FFC Plain or Moisture, and the skin samples were fixed at 2.5 and $5.5 \mathrm{~d}$. Histological sections revealed that the epidermis of 2.5- and 5.5-d-old mice painted with FFC Plain (Figs. 1C, D) or Moisture became thicker than that of control mice (Figs. 1A, B), and contained more numerous keratinocytes (Figs. 1C, D) than that of control mice (Figs. 1A, B). Moreover, the number of mitotic keratinocytes in the epidermis treated with FFC Plain (Figs. 1C, D) or Moisture was greater than in control epidermis (Figs. 1A, B). The same tendency was observed in keratinocytes of developing hair follicles. At $5.5 \mathrm{~d}$, hair follicles in the skin treated with FFC Plain or Moisture were larger than those in control skin. The number of mitotic keratinocytes per epidermis in the control skin gradually decreased after birth, whereas those in the skin treated with FFC Plain or Moisture markedly increased (Fig. 2). The numbers were $3(2.5 \mathrm{~d})$ and 4 (5.5 d) times greater than in control epidermis. No difference was observed between FFC Plain and Moisture. These results suggest that FFC Plain and Moisture stimulate the proliferation of keratinocytes in the epidermis and hair follicles. In addition to the stimulation of keratinocyte proliferation, FFC Plain and Moisture increased the thickness and density of the cornified layer (Fig. 1D), suggesting that FFC Plain and Moisture also stimulate the differentiation of keratinocytes in the epidermis.

The number of fibroblasts in the dermis gradually decreased after birth in the control skin (Fig. 3A), whereas the number of fibroblasts treated with FFC Plain or Moisture increased from 0.5 to $2.5 \mathrm{~d}$ (Fig. $3 \mathrm{~A}, p<0.05$ ) and then decreased. FFC Plain and Moisture almost doubled the number of fibroblasts at 2.5 and $5.5 \mathrm{~d}$. The mitotic index of fibroblasts similarly decreased after birth in the control skin (Fig. 3B), whereas the mitotic index of fibroblasts treated with FFC Plain or Moisture increased from 0.5 to $2.5 \mathrm{~d}$ (Fig. 3B, $p<0.05)$ and then decreased. FFC Plain and Moisture almost doubled $(2.5 \mathrm{~d})$ or tripled $(5.5 \mathrm{~d})$ the mitotic index of fibroblasts. In addition to the stimulation of fibroblast prolifera- 


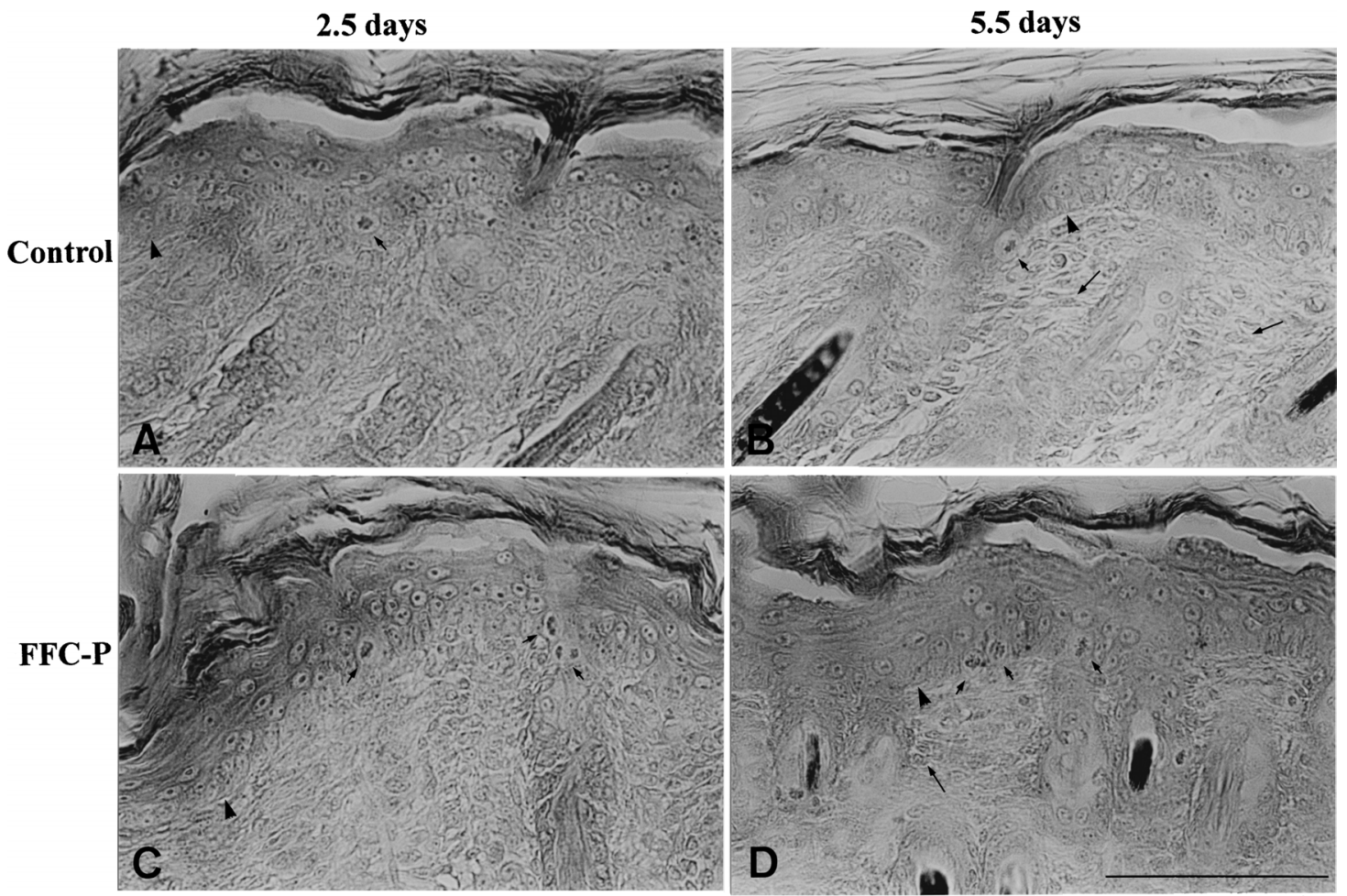

Fig. 1. Effects of FFC on Keratinocytes and Fibroblasts

Histological sections of the dorsal skin of neonatal B10 mice treated with or without FFC Plain. FFC Plain was painted every day on the dorsal skin of B10 mice from $0.5 \mathrm{~d}$ after birth. The dorsal skin of mice treated with (C, D) or without (A, B) FFC Plain were fixed at $2.5 \mathrm{~d}(\mathrm{~A}, \mathrm{C})$ and $5.5 \mathrm{~d}(\mathrm{~B}, \mathrm{D})$. The epidermis painted with FFC Plain (C, D) was thicker than control epidermis (A, B), and the numbers of keratinocytes and mitotic keratinocytes (short arrows) treated with FFC Plain (C, D) were greater than those of control epidermis (A, B). Arrowheads show the basement membrane. Moreover, in the dermis treated with FFC Plain (D, long arrows), fibroblasts increased compared with control dermis (B, long arrows). Scale bar, $100 \mu \mathrm{m}$.

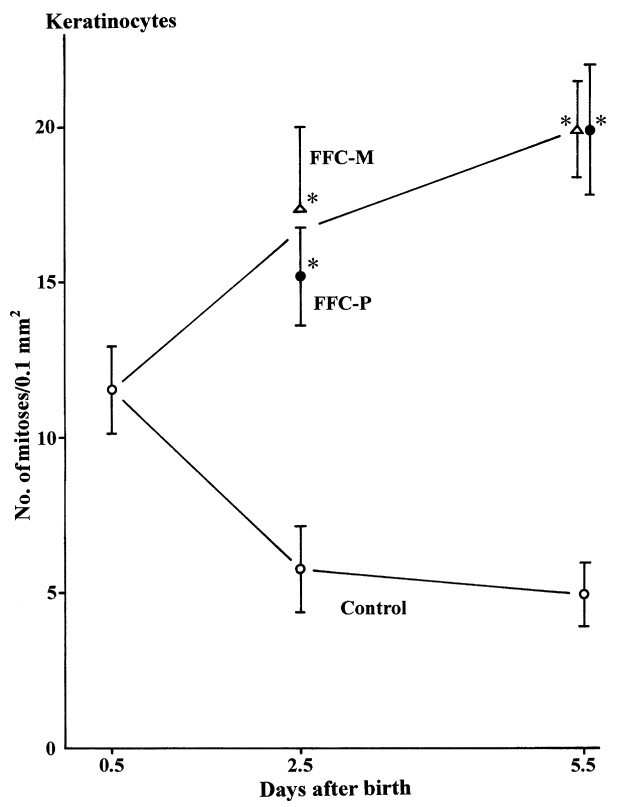

Fig. 2. Effects of FFC on Keratinocyte Proliferation

Changes in the number of mitotic keratinocytes in the epidermis of the dorsal skin of neonatal B10 mice treated with or without FFC Plain or Moisture. The number of mitotic keratinocytes in the control skin gradually decreased after birth. In contrast, its number treated with FFC Plain (FFC-P) and Moisture (FFC-M) increased after birth, and was 3 times higher $(* p<0.05)$ than in the control skin at 2.5 and $5.5 \mathrm{~d}$. No difference in the number of mitotic keratinocytes between FFC Plain and Moisture was observed.

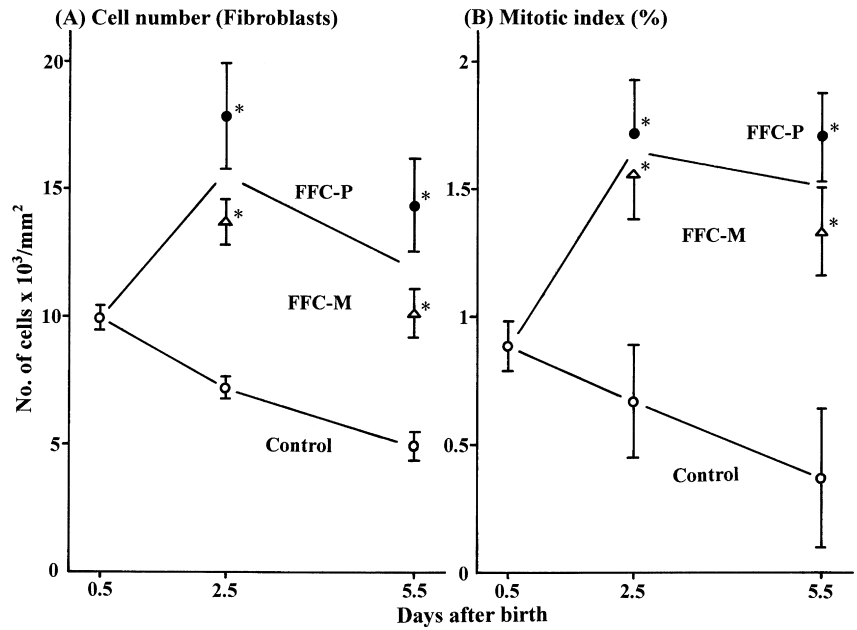

Fig. 3. Effects of FFC on Fibroblast Proliferation

Changes in the number and mitotic index of fibroblasts in the dorsal skin of neonatal B10 mice treated with or without FFC Plain or Moisture. The number of fibroblasts per dermis gradually decreased after birth in control skin (A). However, the number of fibroblasts in the skin treated with FFC Plain (FFC-P) or Moisture (FFC-M) increased from 0.5 to $2.5 \mathrm{~d}(\mathrm{~A}, * p<0.05)$ and then decreased. FFC Plain and Moisture almost doubled the number of fibroblasts 2.5 and $5.5 \mathrm{~d}$ after birth $(* p<0.05)$. Moreover, FFC Plain and Moisture increased the mitotic index of fibroblasts compared with that of the control fibroblasts 2.5 and $5.5 \mathrm{~d}$ after birth $(\mathrm{B}, * p<0.05)$. The mitotic index of fibroblasts gradually decreased after birth in the control skin (B), whereas its index treated with FFC Plain or Moisture increased from 0.5 to $2.5 \mathrm{~d}(\mathrm{~B}, * p<0.05)$, and then decreased. FFC Plain and Moisture almost doubled or tripled the mitotic index of contro fibroblasts $(\mathrm{B}, * p<0.05)$. Although the number of fibroblasts and their mitotic index treated with FFC Moisture are slightly lower than that of FFC Plain, the differences are not statistically significant. 
tion, FFC Plain and Moisture increased the density of fibers around fibroblasts (Fig. 1D), suggesting that FFC Plain and Moisture stimulate the production of fibers, namely the differentiation of fibroblasts. The effects of FFC Plain on the number of fibroblasts and the mitotic index of fibroblasts were slightly greater than those of FFC Moisture, though the differences were not statistically significant.

Effects of FFC Lotions on Melanoblasts and Melanocytes When B10 mice were painted with FFC Plain or Moisture every day, the dorsal skin became darker than control skin. Histochemical observations revealed that in mice treated with FFC Plain (Figs. 4C, D) or Moisture, the number of dopa-positive melanocytes in the epidermis, developing hair follicles, hair bulbs, and dermis were greater than in the control skin (Figs. 4A, B) at $2.5 \mathrm{~d}$ (Figs. 4A, C) and $5.5 \mathrm{~d}$ (Figs. 4B, D). Moreover, in melanocytes treated with FFC Plain (Figs. 4C, D) or Moisture, dendritogenesis, dopamelanin deposition, and cell size were greater than in the control melanocytes (Figs. 4A, B), suggesting that FFC Plain and Moisture stimulate the differentiation of melanocytes through the promotion of tyrosinase activity, melanosome formation, and melanosome transport. ${ }^{7)}$

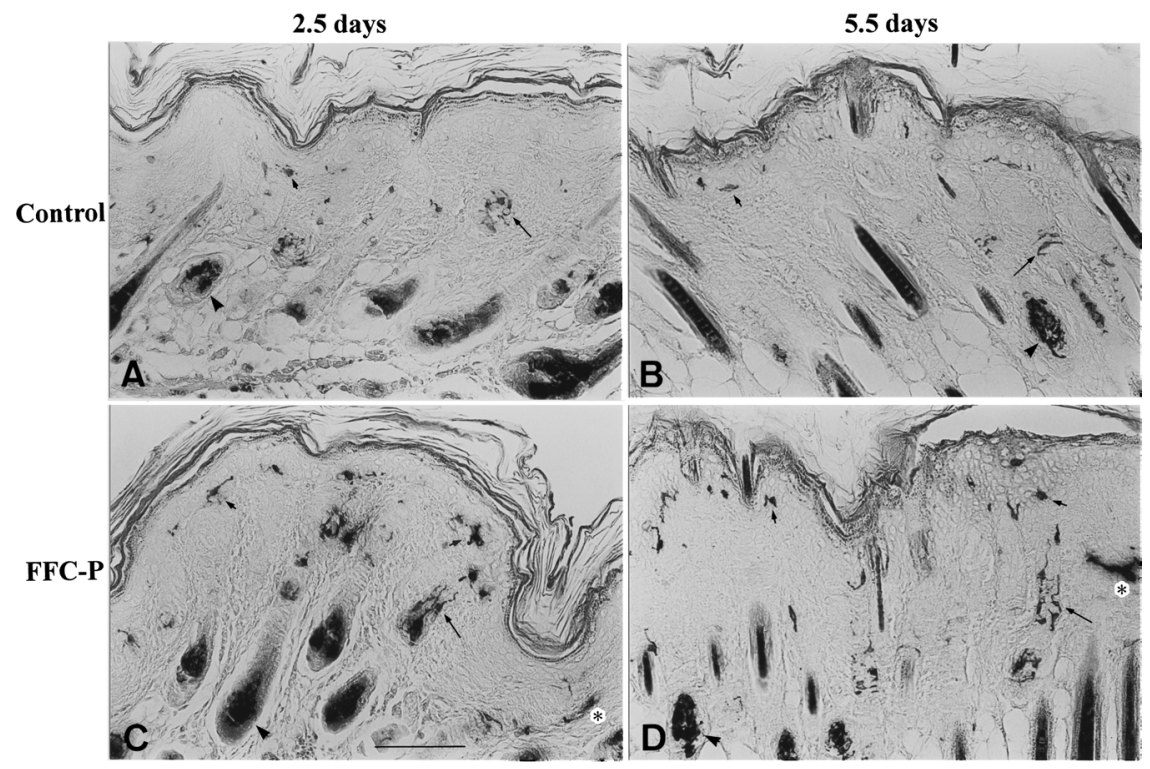

Fig. 4. Effects of FFC on Melanocyte Proliferation and Differentiation

Histochemical (dopa reaction) sections of the dorsal skin of neonatal B10 mice treated with or without FFC Plain. FFC Plain was painted every day on the dorsal skin of B10 mice from $0.5 \mathrm{~d}$ after birth. The dorsal skin of mice treated with (C, D) or without (A, B) FFC Plain was fixed at $2.5 \mathrm{~d}(\mathrm{~A}, \mathrm{C})$ and $5.5 \mathrm{~d}$ (B, D). Dopa-positive melanocytes are observed in the epidermis (short arrows), developing hair follicles (long arrows), hair bulbs (arrowheads), and dermis (asterisks). Melanocytes increased in number in the skin treated with FFC Plain (C, D) compared with the control skin (A, B) at $2.5 \mathrm{~d}(\mathrm{~A}, \mathrm{C})$ and $5.5 \mathrm{~d}(\mathrm{~B}, \mathrm{D})$. In the melanocytes treated with FFC Plain, dendritogenesis, dopa-melanin deposition, and cell size were greater than in the control skin. Scale bar, $100 \mu \mathrm{m}$.

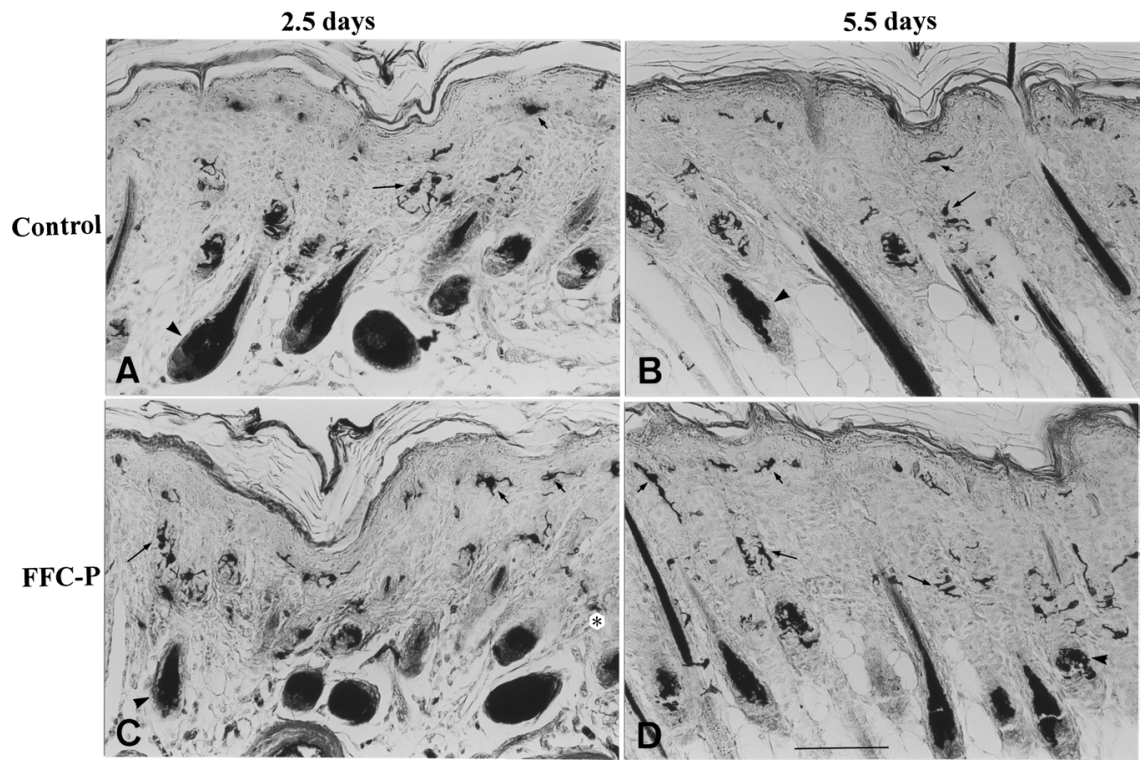

Fig. 5. Effects of FFC on Melanoblast Proliferation

Histochemical (dopa-premelanin reaction) sections of the dorsal skin of neonatal B10 mice treated with or without FFC Plain. The dorsal skin treated with (C, D) or without (A, B) FFC Plain was fixed at $2.5 \mathrm{~d}(\mathrm{~A}, \mathrm{C})$ and $5.5 \mathrm{~d}(\mathrm{~B}, \mathrm{D})$. Cells positive to the combined dopa-premelanin reaction (melanoblasts plus melanocytes) are observed in the epidermis (short arrows), developing hair follicles (long arrows), hair bulbs (arrowheads), and dermis (asterisk). The number of cells positive to the combined dopa-premelanin reaction in the skin treated with FFC Plain (C, D) were greater than in the control skin (A, B) at $2.5 \mathrm{~d}(\mathrm{~A}, \mathrm{C})$ and $5.5 \mathrm{~d}$ (B, D). Experimental protocols are the same as in Fig. 4 . Scale bar, $100 \mu \mathrm{m}$. 
The number of cells positive to the combined dopa-premelanin reactions (melanoblasts plus melanocytes) in the skin treated with FFC Plain (Figs. 5C, D) or Moisture was similarly greater than in the control skin (Figs. 5A, B) at $2.5 \mathrm{~d}$ (Figs. 5A, C) and 5.5 d (Figs. 5B, D). Moreover, in melanoblasts plus melanocytes treated with FFC Plain (Figs. 5C, D) or Moisture, dendritogenesis, silver deposition, and cell size were greater than in the control skin (Figs. 5A, B), suggesting that FFC Plain and Moisture stimulate melanin synthesis, melanosome formation, and melanosome transport.

The number of dopa-positive melanocytes in control epidermis increased after birth. The treatment with FFC Plain or Moisture markedly increased the number of melanocytes at 2.5 and $5.5 \mathrm{~d}$ (Fig. 6A, $p<0.05$ ). FFC Plain and Moisture almost doubled the number of melanocytes at 2.5 and $5.5 \mathrm{~d}$. The number of melanoblasts in the epidermis of B10 mice gradually decreased after birth, as reported previously. ${ }^{8)}$ This decrease was due to the increase in the proportion of melanocytes in the melanoblast-melanocyte population caused by the stimulation of the differentiation of melanoblasts into melanocytes. ${ }^{8)}$ Although the number of melanoblasts treated with FFC Plain or Moisture decreased after birth (Fig. 6B), the number was still greater than in the control skin $(p<0.05)$. The number of melanoblasts plus melanocytes positive to the combined dopa-premelanin reaction in the control epidermis similarly decreased after birth (Fig. 6C). However, the treatment with FFC Plain or Moisture increased the number of melanoblasts plus melanocytes at 2.5 and $5.5 \mathrm{~d}$ (Fig. 6C, $p<0.05$ ). FFC Plain and Moisture almost doubled the number of melanoblasts plus melanocytes at 2.5 and $5.5 \mathrm{~d}$. Moreover, mitoses of melanoblasts and melanocytes were observed in the skin treated with FFC Plain or Moisture. These results suggest that FFC Plain and Moisture stimulate the proliferation of melanoblasts in addition to the stimulation of the proliferation and differentiation of melanocytes in the epidermis.

It is reported that the number of melanocytes in the dermis gradually decreases after birth, as reported previously. ${ }^{15)}$ In this study using B10 mice, the number of melanocytes (Fig. 6D), melanoblasts (Fig. 6E), and melanoblasts plus melanocytes (Fig. 6F) gradually decreased after birth in a similar fashion. However, the treatment with FFC Plain or Moisture inhibited these decreases (Figs. 6D, E, F, $p<0.05$ ). The cell size, dendricity, and silver depositions were increased by the
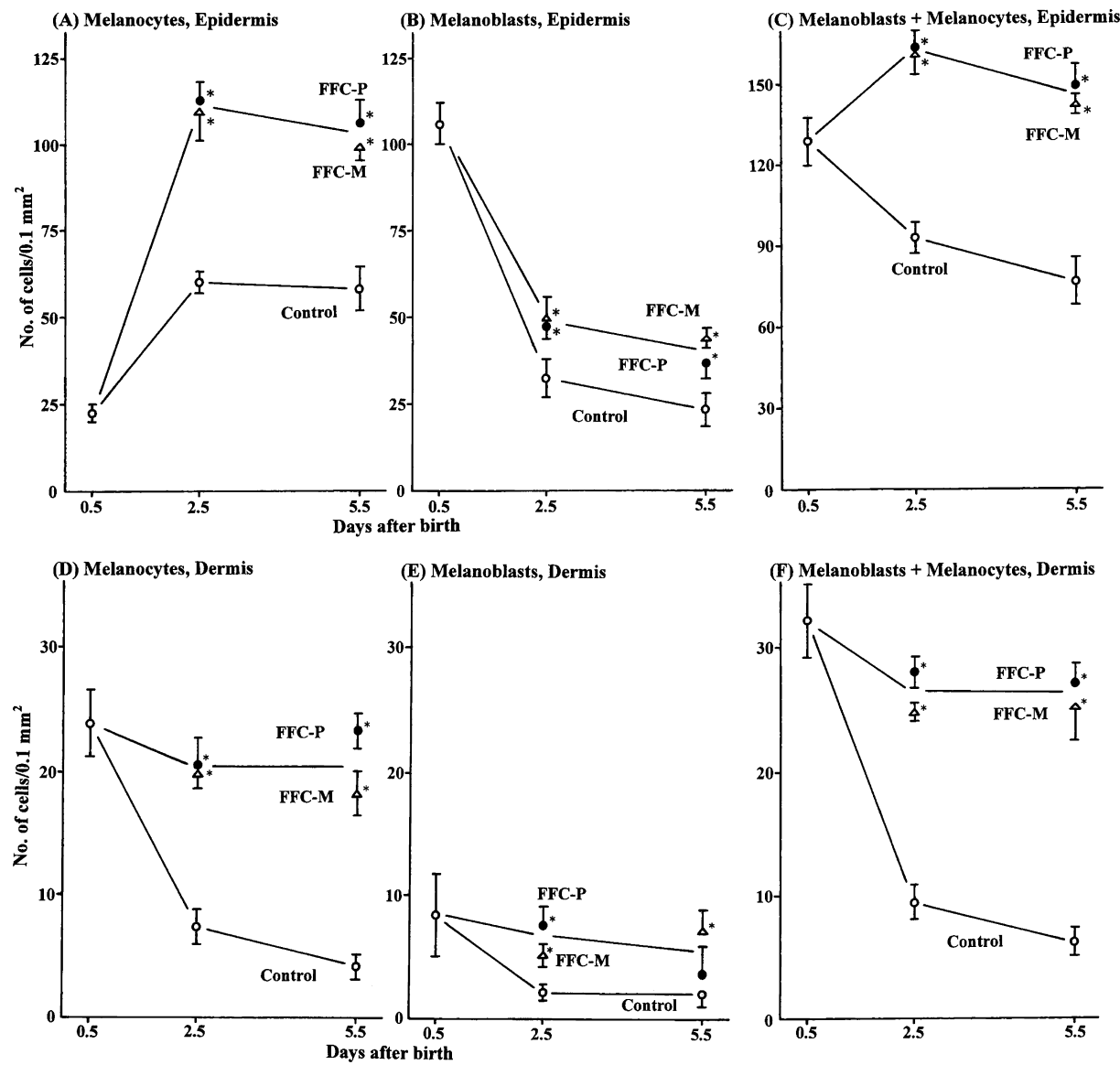

Fig. 6. Effects of FFC on Melanoblasts and Melanocytes

Changes in the number of epidermal (A, B, C) and dermal (D, E, F) melanocytes, melanoblasts, and melanoblasts plus melanocytes in the dorsal skin of neonatal B10 mice treated with or without FFC Plain or Moisture. The number of melanocytes (A) in the control epidermis increased after birth. Treatment of FFC Plain (FFC-P) or Moisture (FFC$\mathrm{M})$ markedly increased the number of melanocytes (A). The number of melanocytes treated with FFC Plain or Moisture was 2-times higher than in the control skin at 2.5 and $5.5 \mathrm{~d}$ $(* p<0.05)$. On the other hand, the number of melanoblasts (B) in the control skin markedly decreased after birth. The number of melanoblasts (B) treated with FFC Plain or Moisture also decreased, but its number is still greater than in the control skin at 2.5 and $5.5 \mathrm{~d}(\mathrm{~B}, * p<0.05)$. The number of melanoblasts plus melanocytes $(\mathrm{C})$ in the control skin similarly decreased after birth, whereas the number treated with FFC Plain or Moisture markedly increased. The number of melanoblasts plus melanocytes is 2-times higher than that of control skin at 2.5 and $5.5 \mathrm{~d}(* p<0.05)$. Although the numbers of epidermal melanocytes and melanoblasts plus melanocytes treated with FFC Moisture are slightly lower than those of FFC Plain, the differences are not statistically significant. The number of dermal melanocytes (D), melanoblasts (E), and melanoblasts plus melanocytes (F) gradually decreased after birth. However, the treatment with FFC Plain or Moisture inhibited these decreases $(\mathrm{D}, \mathrm{E}, \mathrm{F}, * p<0.05)$. Although the numbers of dermal melanocytes and melanoblasts plus melanocytes treated with FFC Moisture are slightly lower than those of FFC Plain, the differences are not statistically significant. 
treatment of FFC Plain or Moisture. Moreover, mitoses of dermal melanoblasts and melanocytes were observed in the skin treated with FFC Plain or Moisture. These results suggest that FFC Plain and Moisture stimulate the proliferation and differentiation of melanoblasts and melanocytes in the dermis, and inhibit the decrease in the number of dermal melanocytes after birth in a similar fashion to the epidermis.

Effects of FFC Lotions on Hair Growth and Hair-Loss Treatment of the skin with FFC Plain or Moisture stimulated the development of hair at $5.5 \mathrm{~d}$ (Figs. 4D, 5D) and $6.5 \mathrm{~d}$ (Fig. 7A). At $14.5 \mathrm{~d}$, the hair in the mice treated with FFC Plain (Fig. 7B) or Moisture was darker and more lustrous than the control hair. It is known that B10 mice generally

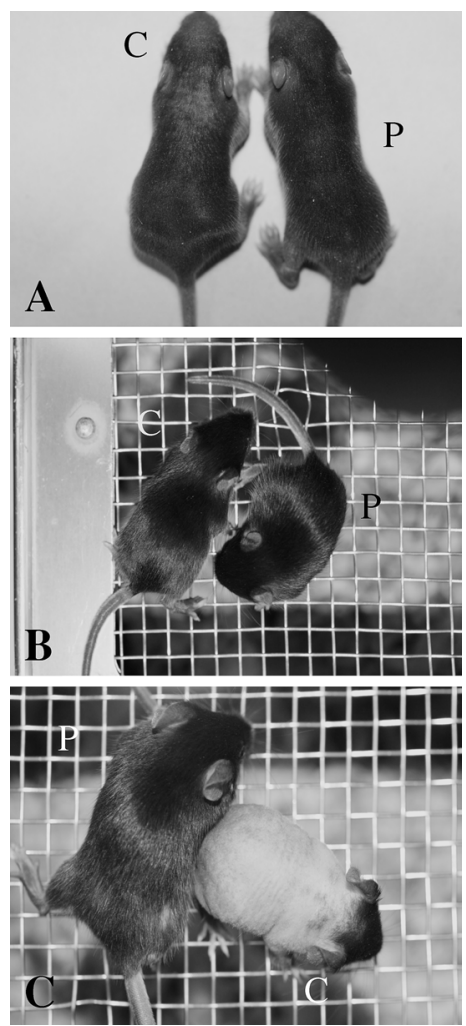

Fig. 7. Effects of FFC on Hair Growth and Hair-Loss

Effects of FFC Plain on hair growth and the alopecia hair-loss in neonatal B10 mice. (A) In FFC Plain-treated mice (P), hair grew faster than in control mice (C) at $6.5 \mathrm{~d}$. (B) At $14.5 \mathrm{~d}$, the hair treated with FFC-Plain (P) was darker and more lustrous than control hair (C). (C) B10 mice generally lose their hair at the telogen stage owing to the alopecia hair-loss. In most B10 mice, cutaneous hairs except those on the head are completely lost from 2 to 3 weeks after birth $(19.5 \mathrm{~d}, \mathrm{C})$. However, in mice treated with FFC Plain (P) showed almost no hair-loss. lose their hair at the telogen stage of the hair growth cycle. This hair-loss is caused by the expression of the alopecia gene. ${ }^{16)}$ In most B10 mice, some or all cutaneous hairs except those on the head are lost from 2 to 3 weeks after birth (Fig. 7C). Regrowth of hair initiates after 3 to 4 weeks, and complete cutaneous hairs are recovered. In the following telogen stages of the hair growth cycle, the hair-loss by the alopecia occurs, but the area of hair-loss decreases as age advances. In this study, all mice in control group possessed alopecia hair-loss (Fig. 7C, Table 1). However, in mice painted with FFC Plain (Fig. 7C) or Moisture, almost no hair-loss was observed at $19.5 \mathrm{~d}$, suggesting that FFC lotions inhibit the alopecia hair-loss. As shown in Table 1, in mice treated with FFC Plain, the percentage of mice with no hairloss (no alopecia expression) was more than $72 \%$, about $17 \%$ mice showed slight hair-loss, about $11 \%$ had lost half their hair, and no complete hair-loss was observed. On the other hand, in mice treated with FFC Moisture, the percentage of mice with no hair-loss was only $44 \%$, about $28 \%$ showed slight hair-loss, about $28 \%$ had lost half hair, and no complete hair-loss was observed. Thus, the inhibition of the alopecia by FFC Moisture was weaker than by FFC Plain. Moreover, the treatment with FFC Plain or Moisture delayed hair-loss by 1 or $2 \mathrm{~d}$ (Table 1), suggesting that even in mice in which the alopecia hair-loss failed to be completely inhibited, the proceeding of the hair-loss is delayed.

\section{DISCUSSION}

The author's previous study showed that FFC stimulated the proliferation of cultured keratinocytes, melanoblasts, and melanocytes in newborn mice. ${ }^{4)}$ FFC also stimulated the proliferation of cultured fibroblasts derived from newborn mice (Hirobe, unpublished). FFC also stimulated the differentiation of murine keratinocytes and melanocytes. ${ }^{5)}$ Moreover, FFC stimulated the proliferation of cultured normal human keratinocytes, fibroblasts, and melanocytes. ${ }^{6}$ ) The present findings in vivo confirm the author's previous studies using murine and human skin cells in vitro. Considering all these findings, it is conceivable that FFC can stimulate the proliferation of mouse and human keratinocytes, melanocytes, and fibroblasts. FFC was also found to stimulate hair growth and to inhibit the alopecia hair-loss in this study. Since the hair growth is regulated by the proliferation and differentiation of keratinocytes, melanocytes, and fibroblasts, ${ }^{7)}$ it is reasonable to think that FFC is involved in the homeostasis of the skin and hair by regulating the proliferation and differentiation of

Table 1. Effects of FFC Plain and Moisture on the Alopecia Hair-Loss in B10 Mice

\begin{tabular}{|c|c|c|c|c|}
\hline Treatment & - & + & ++ & +++ \\
\hline Control & 0 & 0 & $\begin{array}{l}14.3 \%(+4: \delta 2) \\
17.3 \mathrm{~d}\end{array}$ & $\begin{array}{l}85.7 \%(\text { ( } 17: \text { ठ } 19) \\
17.0 \mathrm{~d}\end{array}$ \\
\hline FFC Plain & $72.2 \%($ ( $6: \delta 7)$ & $\begin{array}{l}16.7 \%(90: \text { ठ3 }) \\
19.5 \mathrm{~d}\end{array}$ & $\begin{array}{l}11.1 \%\left(+2: \delta^{\dagger} 0\right) \\
19.5 \mathrm{~d}\end{array}$ & 0 \\
\hline FFC Moisture & $44.4 \%(+5: ð 3)$ & $\begin{array}{l}27.8 \%(\uparrow 3: ठ 2) \\
18.9 \mathrm{~d}\end{array}$ & $\begin{array}{l}27.8 \%(q 1: \delta 4) \\
17.5 \mathrm{~d}\end{array}$ & 0 \\
\hline
\end{tabular}

The dorsal skin of 0.5-d-old B10 mice was painted with or without (Control) FFC Plain or Moisture four times a week. The expression of the alopecia was carefully observed in all mice. - : no alopecia expression (complete hair, the number of female and male is written), + : slight hair-loss, $++:$ half hair-loss, $+++:$ complete hair-loss except those on the head (complete alopecia expression). Data show the average percentage of mice with alopecia and date in which maximal alopecia was observed. For example, "FFC Moisture $18.9 \mathrm{~d}$ " means that the mice treated with FFC Moisture showed slight hair-loss at $21 \mathrm{~d}$ and the hair-loss already reached on a maximal level at $18.9 \mathrm{~d}$ (in average of 5 mice) and then kept on this level until $21 \mathrm{~d}$. 
keratinocytes, melanocytes, and fibroblasts.

In the present study, FFC Plain was more effective than Moisture in stimulating the proliferation of fibroblasts and melanocytes, and in inhibiting the alopecia. Although the reason why FFC Moisture is less effective than Plain cannot be fully explained at present, it might be due to the inhibitory effect of rose oil, grape seed oil, jojoba oil, and the emulsifiers present in Moisture.

The proliferation and differentiation of mammalian keratinocytes are regulated by numerous factors, including epidermal growth factor (EGF), ${ }^{17}$ keratinocyte growth factor $(\mathrm{KGF}),{ }^{18)}$ hydrocortisone, ${ }^{19)}$ and dexamethasone. ${ }^{19)}$ The proliferation and differentiation of mammalian fibroblasts are regulated by basic fibroblast growth factor (bFGF) ${ }^{20)}$ acidic FGF (aFGF), ${ }^{20)}$ transforming growth factor $\beta_{1},{ }^{21)} \mathrm{KGF}{ }^{21)}$ interleukin (IL)- $1 \alpha,{ }^{21)} \mathrm{IL}-1 \beta,{ }^{21)}$ and platelet-derived growth factor. $^{22)}$ Moreover, the proliferation and differentiation of mammalian melanocytes are regulated by melanocyte-stimulating hormone, ${ }^{10,13,23,24)}$ endothelin (ET)-1, ${ }^{25-27)}$ ET-2, ${ }^{27)}$ ET$3,{ }^{27)} \mathrm{bFGF}^{28)}$ steel factor, ${ }^{29,30)}$ leukemia inhibitory factor, ${ }^{31)}$ granulocyte-macrophage colony-stimulating factor, ${ }^{32,33)}$ and hepatocyte growth factor (HGF). ${ }^{34,35)}$ Mammalian hair growth is also stimulated by insulin-like growth factor- $\mathrm{I},{ }^{36}$ ) $\mathrm{HGF}^{37)}$ RET tyrosine kinase, ${ }^{38)}$ bone morphogenetic protein, ${ }^{39)}$ estrogen, ${ }^{40)}$ macrophage-stimulating protein, ${ }^{41)}$ FGF$18,{ }^{42}$ and nerve growth factor. ${ }^{43)}$ It should be emphasized that FFC can stimulate the proliferation and differentiation of keratinocytes, melanocytes, and fibroblasts as well as hair growth without adding these growth factors, cytokines, and hormones to skin lotions. FFC may activate these factors and stimulate signaling pathways ${ }^{44-46)}$ elicited by these factors. Furthermore, it is possible that FFC changes the homeostatic conditions within the skin and hair cells to those favorable for the stimulation of proliferation and differentiation.

It is unknown at present what factors cause alopecia in B10 mice. The growth factors and cytokines involved in regulating the proliferation and differentiation of the three cell lineages in the skin might be defective in the alopecia mice. It may be possible that FFC treatment restores the defects in the expression of the genes associated with the proliferation and differentiation of keratinocytes, fibroblasts, and melanocytes. However, this hypothesis remains to be investigated in a future study.

In the present study, the proliferation and differentiation of keratinocytes, fibroblasts, and melanocytes were stimulated by FFC. Thus, FFC seems to affect these growth factors, cytokines and hormones in vivo, and bring the changes of renewal of 3 cell types that constitute skin and hair followed by the homeostasis of skin and hair. Stimulation of the proliferation and differentiation of keratinocytes, fibroblasts, and melanocytes by FFC may be helpful in maintaining the health and youthfulness of the skin and hair. Thus, it is reasonable to think that FFC can maintain the health and youthfulness of skin and hair through the activation of the proliferation and differentiation of keratinocytes, fibroblasts, and melanocytes.

Acknowledgments The author thanks Dr. H. Kunoh, Dr. T. Nishimura, Mr. H. Tagata, Mr. M. Iinomi, and Miss H. Mizuno of the Institute for Biological Process Research of Akatsuka Co., Mie, Japan for valuable discussions and for generous supply of FFC Plain and Moisture.

\section{REFERENCES}

1) Sugi J., Yamashita S., Bull. Soc. Sea Water Sci. Japan, 42, 11 (1991).

2) Hasegawa S., Meguro A., Shimizu M., Nishimura T., Kunoh H., Actinomycetologica, 20, 23-29 (2006).

3) Brain J., "Abst. Anti-aging Int. Symp. Expo. Tokyo 2006," p. 14 (2006).

4) Hirobe T., J. Health Sci., 53, 576-584 (2007).

5) Hirobe T., J. Health Sci., 55, 86-94 (2009).

6) Hirobe T., J. Health Sci., 55, 447-455 (2009).

7) Hirobe T., Histol. Histopathol., 10, 223-237 (1995).

8) Hirobe T., Anat. Rec., 208, 589-594 (1984).

9) Mishima Y., J. Invest. Dermatol., 34, 355-360 (1960).

10) Hirobe T., Takeuchi T., J. Embryol. Exp. Morphol., 37, 79-90 (1977).

11) Mishima Y., J. Histochem. Cytochem., 12, 784-790 (1964).

12) Hirobe T., J. Exp. Zool., 224, 355-363 (1982).

13) Hirobe T., Pigment Cell Res., 5, 1-11 (1992).

14) Zembowicz A., Mihm M. C., Histopathology, 45, 433-451 (2004).

15) Tamate H. B., Hirobe T., Takeuchi T., J. Exp. Zool., 238, 235-240 (1986).

16) Sundberg J. P., Shultz L. D., J. Invest. Dermatol., 96, 95S-96S (1991).

17) Rheinwald J. G., Green H., Nature (London), 265, $421-424$ (1977)

18) Rubin J. S., Osada H., Finch P. W., Taylor W. G., Rudikoff S., Aaronson S. A., Proc. Natl. Acad. Sci. U.S.A., 86, 802-806 (1989).

19) Hirobe T., Dev. Biol., 161, 59-69 (1994).

20) Tamm I., Kikuchi T., Zychlinsky A., Proc. Natl. Acad. Sci. U.S.A., 88 3372-3376 (1991).

21) Maas-Szabowski N., Shimotoyodome A., Fusenig N. E., J. Cell Sci., 112, 1843-1853 (1999).

22) Li W., Fan J., Chen M., Guan S., Sawcer D., Bokoch G. M., Woodley D. T., Mol. Biol. Cell, 15, $294-309$ (2004).

23) Thody A. J., Ridley K., Penny R. J., Chalmers R., Fisher C., Shuster S., Peptides, 4, 813-816 (1983)

24) Schauer E., Trautinger F., Koeck A., Schwarz A., Bhardwai R., Simon M., Ansel J. C., Schwarz T., J. Clin. Invest., 93, 2258-2262 (1994).

25) Imokawa G., Kobayashi T., Miyagishi M., Higashi K., Yada Y., Pig ment Cell Res., 10, 218-228 (1977).

26) Tada A., Suzuki I., Im S., Davis M. B., Cornelius J., Babcock G. Nordlund J., Abdel-Malek Z. A., Cell Growth Differ., 9, 575-584 (1998).

27) Hirobe T., J. Invest. Dermatol. Symp. Proc., 6, 25-31 (2001).

28) Halaban R., Langdon R., Birchall N., Cuono C., Baird A., Scott G. Moellmann G., McGuire J., J. Cell Biol., 107, 1611-1619 (1988).

29) Kunisada T., Yoshida H., Yamazaki H., Miyamoto A., Hemmi H. Nishimura E., Shultz L. D., Nishikawa S.-I., Hayashi S.-I., Development, 125, 2915-2923 (1998).

30) Hirobe T., Osawa M., Nishikawa S.-I., Pigment Cell Res., 16, 644 655 (2003).

31) Hirobe T., J. Cell. Physiol., 192, 315-326 (2002)

32) Imokawa G., Yada Y., Kimura M., Morisaki N., Biochem. J., 313 625-631 (1996).

33) Hirobe T., Furuya R., Ifuku O., Osawa M., Nishikawa S.-I., Exp. Cell Res., 297, 593-606 (2004).

34) Kunisada T., Yamazaki H., Hirobe T., Kamei S., Omoteno M., Tagaya H., Hemmi H., Koshimizu U., Nakamura T., Hayashi S.-I., Mech. Dev., 94, 67-78 (2000).

35) Hirobe T., Osawa M., Nishikawa S.-I., Pigment Cell Res., 17, 51-61 (2004).

36) Su H.-Y., Hickford J. G. H., The P. H. B., Hill A. M., Frampton C. M., Bickerstaffe R., J. Invest. Dermatol., 112, 245-248 (1999).

37) Lindner G., Menrad A., Gherardi E., Merlino G., Welker P., Handjiski B., Roloff B., Paus R., FASEB J., 14, 319-332 (2000).

38) Kato M, Takeda K., Kawamoto Y., Tsuzuki T., Dai Y., Nakayama S., Toriyama K., Tamada Y., Takahashi M., Nakashima I., Oncogene, 20, 7536 - 7541 (2001)

39) Kobielak K., Pasolli H. A., Alonso L., Polak L., Fuchs E., J. Cell Biol., 163, 609-623 (2003).

40) Ohnemus U., Unalan M., Handjiski B., Paus R., J. Invest. Dermatol. 122, 7-13 (2004).

41) McElwee K. J., Huth A., Kissling S., Hoffmann R., J. Invest. Dermatol., 123, $34-40$ (2004).

42) Kawano M., Komi-Kuramochi A., Asada M., Suzuki M., Oki J., Jiang J., Imamura T., J. Invest. Dermatol., 124, 877-885 (2005).

43) Peters E. M. J., Hendrix S., Golz G., Klapp B. F., Arck P. C., Paus R., J. Histochem. Cytochem., 54, 275-288 (2006).

44) Coughlin S. R., Barr P. J., Cousens L. S., Fretto L. J., Williams L. T., J. Biol. Chem., 263, 988-993 (1988)

45) Imokawa G., Pigment Cell Res., 17, 96-110 (2004).

46) Hirobe T., Pigment Cell Res., 18, 2-12 (2005). 